\title{
A retrospective comparative study of open reduction and cannulated screw fixation and Kirschner wire fixation in the treatment of fracture of lateral condyle of humerus in children
}

\author{
XIANYONG LUO \\ Zhengzhou University First Affiliated Hospital \\ XINRANG CHEN \\ Zhengzhou University First Affiliated Hospital \\ JIAXIANG WANG ( $\nabla$ wjiaxiang08@126.com ) \\ Zhengzhou University First Affiliated Hospital
}

\section{Research Article}

Keywords: Lateral condylar fractures of the humerus, Cannulated screw, Kirschner wire, Prognosis

Posted Date: February 24th, 2021

DOI: https://doi.org/10.21203/rs.3.rs-165597/v1

License: (c) (1) This work is licensed under a Creative Commons Attribution 4.0 International License.

Read Full License 


\section{Abstract}

Objective To evaluate the clinical efficacy of two surgical methods in the treatment of fracture of lateral condyle of humerus in children and the application value of two kinds of internal fixation materials.

Methods A total of 32 children with fracture of lateral condyle of humerus were treated in our hospital from June 2015 to June 2018, with an average age of 5.6 years, including 18 males and 14 females. The patients were divided into two groups: group $A(n=12)$ and group $B(n=20)$. By comparing the operation time, removal of internal fixation time, Dhillon elbow function evaluation, HHS elbow function score, X-ray measurement of postoperative carrying angle, Baumann angle and distal humerus anteversion angle, the operation-related complications were recorded in detail.

Results The operation time was $0.9 \pm 0.2$ hours in group $A, 0.8 \pm 0.2$ hours in group $B, P>0.05$. The removal internal fixation time was $91 \pm 8$ days in group $A, 59 \pm 10$ days in group $B(P<0.05)$. The Dhillon elbow function score was $8.2 \pm 0.6$ in group $A, 8.0 \pm 0.8$ in group $B$, and $93 \pm 5$ in group $A$ and $89 \pm 4$ in group $B(P>0.05)$. The carrying angle was $10^{\circ} \pm 1^{\circ}$ in group $A$ and $10^{\circ} \pm 2^{\circ}$ in group $B(P>0.05)$. The Baumann angle was $15^{\circ} \pm 4^{\circ}$ in group $A$ and $16^{\circ} \pm 3^{\circ}$ in group $B(P>0.05)$. The anteversion of distal humerus was $33^{\circ} \pm 3^{\circ}$ in group $A$ and $33^{\circ} \pm 4^{\circ}$ in group $B(P>0.05)$. In the last follow-up, the excellent and good rate of elbow joint function in group $A$ was excellent in 8 cases and good in 4 cases, and the excellent and good rate in group B was 100\%. In group B, 13 cases were excellent, 6 cases were good, and 1 case was moderate, with an excellent and good rate of $95 \%$. The postoperative complications were observed in group A, including avascular necrosis of lateral condyle in 1 case, premature closure of epiphysis in 1 case, skin irritation in 5 cases, needle exudation and incision infection in 2 cases, hyperplasia of lateral condyle in 3 cases, and loss of extension and flexion in 1 case.

Conclusions There was no significant difference in operation time, elbow function recovery and imaging measurement between the two surgical methods in the treatment of lateral condylar fracture of humerus. Kirschner wire group had a certain advantage over cannulated cancellus screw group in fracture healing and removal of internal fixation. however, the clinical observation screw group has advantages in postoperative nursing and early exercise.

\section{Introduction}

Fracture of lateral condyle of humerus in children is a common fracture of elbow joint in children, and it is also the second most common fracture of elbow. Due to the difference of medical level in different regions, doctors have different diagnosis and treatment methods for this kind of fracture, and children may have different prognosis. This fractures have a common complications including nonunion, malunion, ulnar nerve paresis, hypertrophic scar, avascular necrosis of ossific nucleus and angular deformity. At present, the opinions on the treatment of fractures of lateral condyle of humerus are basically the same. It is considered that fractures with displacement $\leq 2 \mathrm{~mm}$ can be treated 
conservatively, while fractures with displacement $>2 \mathrm{~mm}$ should be treated by operation. ${ }^{1}$ In terms of surgical treatment, the choice of internal fixation materials is different. The commonly used methods of internal fixation include Kirschner wire and screw fixation, however, the prognosis seems to vary regardless of the treatment. This study group compared the clinical effects of cannulated cancellus screw and Kirschner wire in the treatment of fracture of lateral condyle of humerus in children, and summarized the differences in postoperative recovery and complications of the two internal fixation methods.

\section{Materials And Methods}

The study design was retrospective, nonrandomized, comparative study. Institutional ethical clearance and informed patient consent were obtained before enrolment of patients for the study.

\section{General information}

A total of 32 children with fracture of lateral condyle of humerus were treated in our hospital from June 2015 to June 2018, with an average age of 5.6 years (range from 3 to 8 years). There were 18 males and 14 females. All the patients underwent X-ray films of the anterior and lateral position of the elbow joint when they were admitted to the hospital, and the fractures with displacement of $2 \mathrm{~mm}$ or left or right were diagnosed by CT scan of the elbow joint. According to Jacob classification, ${ }^{2}$ there were 18 cases of type II and 14 cases of type III. All children in this group were treated with open operation after routine examination after admission and exclusion of surgical contraindications. The time from injury to operation was 2-8 days, with an average of 3.5 days. Exclusion criteria: (1) cases treated for more than one week after injury, and (2) cases fixed with Kirschner wire after closed reduction. According to the choice of internal fixation materials, the patients with open reduction were divided into group $A(n=12)$ and group $B(n=20)$.

\section{Treatment methods}

All the children were treated with general intravenous anesthesia, including 5 cases with brachial plexus combined anesthesia, upper axillary tourniquet before operation, Kocher elbow lateral incision through the space between brachioradialis and triceps brachii to periosteum, avoiding radial nerve, opening periosteum to expose fracture end, cleaning congestion, reduction of fracture with reduction forceps or cloth towel forceps under direct vision, according to the size of fracture block, whether there was lateral epicondyle bone. The broken end of the fracture was fixed with cannulated cancellus screw(partial thread) or Kirschner wire. Group A was fixed with cannulated cancellus screws with two screws, $3.0 \mathrm{~mm}$ or $3.5 \mathrm{~mm}$ in diameter, screws are passing in the metaphyseal portion of fracture without passing through the capitulum humeri, crossed or parallel in direction, but contralateral cortex. Group B was fixed with Kirschner wire and selected two or three Kirschner wires with a diameter of $1.5 \mathrm{~mm}$ or $2.0 \mathrm{~mm}$, which could pass through the capitulum humeri if the metaphyseal portion of fracture is small, spread out in a fan shape in the direction and pass through the contralateral cortex. Typical cases are shown in Figs. 1 and 2. 
During the operation, the reduction and fixation of the fracture was completed under C-arm fluoroscopy to ensure the anatomical reduction of the broken end of the fracture. In group $B$, the needle tail was bent and placed in the skin as far as possible. After the completion of internal fixation, mild flexion and extension of the elbow joint was performed to judge whether the fracture block was stable or not. after washing and hemostasis, the lateral supporting ligament was sutured intermittently and the skin was sutured intradermally. All the children in this group were fixed with $90-110^{\circ}$ upper limb plaster.

Figure 1: male, 5 years old, Jacoblll type, with open reduction and cannulated cancellus screw fixation.

\section{Evaluation of curative effect}

The operation time of this group of cases was recorded in detail. through postoperative follow-up, regular X-ray examination of the anterior and lateral position of the elbow joint was performed to understand the fracture healing, the time of removal of internal fixation and the range of flexion and extension of the elbow joint. Dhillon elbow function evaluation and HHS elbow function score were used to evaluate the postoperative treatment effect of the children. ${ }^{3}$ The elbow lifting angle, Baumann angle and anteversion angle of distal humerus measured by X-ray in the last follow-up were compared.

\section{Statistical processing}

The measurement data were expressed by _x $\mathrm{x}$, and the data were statistically analyzed by SPSS19.0 statistical software, and the difference was considered to be statistically significant by $t$-test.

\section{Results}

According to the operation records, the operation time in group A was $0.6 \pm 1.3 \mathrm{~h}$, with an average of $0.9 \pm$ $0.2 \mathrm{~h}$. In group $\mathrm{B}$, the operation time was $0.5 \pm 1.1 \mathrm{~h}$, with an average of $0.8 \pm 0.2 \mathrm{~h}$. Regular $\mathrm{X}$-ray imaging examination was performed to judge the primary healing of the fracture through the blurred fracture line and the formation of new bone around the fracture. All the children in this group were fixed with plaster after operation, and some of them were protected by brace after 2 weeks. All the children in group A were treated with elbow joint function exercise after 4 weeks. The children in group B included subcutaneous indwelling at the end of needle in 16 cases, extradermal indwelling in 4 cases, wearing plaster or brace for more than 4 weeks. The protective time for those exposed to the skin with Kirschner needle reached 6 weeks. Except for 4 cases of early Kirschner needle removal in group B, two-stage incision and internal fixation was performed when the joint function reached or close to normal. The internal fixation time was $75 \pm 106$ days in group $A$, with an average of $91 \pm 8$ days in group $B$ and $42 \pm 80$ days in group $B$, with an average of $59 \pm 10$ days.

All cases were followed up for $0.8 \sim 3.5$ years (mean $2.2 \pm 0.8$ years). All the 32 children had primary fracture healing, and no re-displacement, nonunion and nerve injury were found. The postoperative follow-up Dhillon elbow function score was $8.2 \pm 0.6,8.0 \pm 0.8,93 \pm 5$ in group $A n$ and $89 \pm 4$ in group $B, P$ $>0.05$. The elbow function scores of group $A n$ and group $B$ were $93 \pm 5$ and $89 \pm 4$ respectively. The last $X$ - 
ray measurement showed that the lifting angle was $10^{\circ} \pm 1^{\circ}$ in group $A n$ and $10^{\circ} \pm 2^{\circ}$ in group $B(P>0.05)$. The Baumann angle was $15^{\circ} \pm 4^{\circ}$ in group $A n$ and $16^{\circ} \pm 3^{\circ}$ in group $B(P>0.05)$. The anteversion of distal humerus was $33^{\circ} \pm 3^{\circ}$ in group $A n$ and $33^{\circ} \pm 4^{\circ}$ in group $B(P>0.05)$. According to the Dhillon score standard, 9 points were excellent, 7-8 points were good, 5-6 scores were medium, less than 5 points were poor, group A was excellent in 8 cases, good in 4 cases, excellent and good rate was $100 \%$ in group B, excellent in 13 cases, good in 6 cases, fair in 1 case, excellent and good rate of $95 \%$. The statistical comparison among the groups is shown in Table 1:

Table 1

Statistical comparison of data between group A and group B

\begin{tabular}{|llll|}
\hline & group A & group B & P \\
\hline the operation time(h) & $0.9 \pm 0.2 \mathrm{~h}$ & $0.8 \pm 0.2 \mathrm{~h}$ & $\mathrm{P}=0.65$ \\
removal of internal fixation time(d) & $91 \pm 8 \mathrm{~d}$ & $59 \pm 10 \mathrm{~d}$ & $\mathrm{P}=0.00$ \\
Dhillon elbow function evaluation & $8.2 \pm 0.6$ & $8.0 \pm 0.8$ & $\mathrm{P}=0.31$ \\
HHS elbow function score & $93 \pm 5$ & $89 \pm 4$ & $\mathrm{P}=0.08$ \\
measurement of postoperative carrying angle $\left(^{\circ}\right)$ & $10 \pm 1$ & $10 \pm 2$ & $\mathrm{P}=0.93$ \\
Baumann angle $\left(^{\circ}\right)$ & $15 \pm 4$ & $16 \pm 3$ & $\mathrm{P}=0.55$ \\
distal humerus anteversion angle $\left(^{\circ}\right)$ & $33 \pm 3$ & $33 \pm 4$ & $\mathrm{P}=0.83$ \\
\hline
\end{tabular}

Statistics of postoperative complications: group An included 1 case of ischemic necrosis of lateral condyle and 1 case of premature closure of epiphysis. Follow-up showed that the appearance deformity was not obvious and the functional activity was normal. In group B, there were 5 cases of skin irritation with bursitis, of which 1 case broke through the end of Kirschner needle to the skin, and the symptoms were relieved after internal fixation. There were 2 cases of needle exudation and incision infection, both of which were left out of the skin at the end of the needle, and the local scar healed by dressing change after needle extraction. There were 3 cases of lateral condyle hyperplasia with normal functional activity, 1 case lost $10^{\circ}$ extension and $15^{\circ}$ flexion.

\section{Discussion}

Fractures of the lateral condyle of humerus (lateralcondylarfracturesofthehumerus,LCF) in children are the most common elbow fractures involving the growth plate, accounting for $12-20 \%$ of all upper limb fractures in children. These fractures most commonly occur in children between 4 and 10 years old, with the highest incidence in children around 6 years old. ${ }^{4}$ The common injury mechanism leading to fracture is supination of the forearm in the straight position when falling, and varus violence leads to traction of the extensor tendon and brachioradialis muscle of the forearm (traction mechanism, pull-off). Other injury mechanisms include fracture of the lateral condyle caused by elbow valgus impact (push-out mechanism, push-off) and direct injury of the elbow. ${ }^{5}$ As we all know, missed diagnosis or delayed 
diagnosis and treatment of LCF may lead to joint stiffness, fracture nonunion, followed by serious cubitus valgus, delayed ulnar nerve paralysis and other complications. At present, we have basically reached a consensus on the diagnosis and treatment of LCF, but there are still some disputes.

\section{I. selection of surgical indications and surgical methods}

At present, the treatment of LCF is mainly based on the specific classification of fracture, the commonly used types are: 1 Milch type, according to whether the pulley joint surface is involved into type 2, 2Jakob type, according to whether the cartilage hinge is broken and the degree of displacement is divided into 3 types. Milch type I can be considered as a variant of Salter- Harris type IV epiphyseal injury, while Milch type II is equivalent to Salter- Harris type II epiphyseal injury. ${ }^{6}$

At present, the research of evidence-based medicine thinks that the degree of $\mathrm{X}$-ray fracture displacement can be used as the best basis for guiding operation and clinical decision. According to fracture

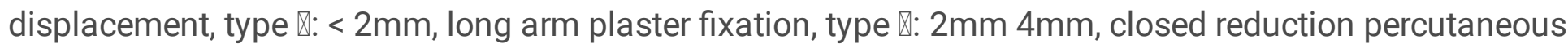

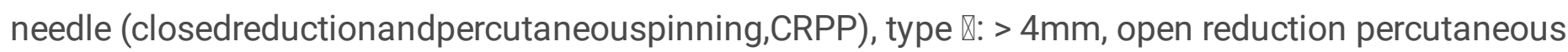
needle (openreductionandpercutaneouspinning,ORPP). ${ }^{7}$ However, there are still controversial areas, such as Song et al. ${ }^{8}$ think that closed reduction and internal fixation is an effective method for the treatment of unstable displacement of LCF in children. If the fracture displacement is more than $2 \mathrm{~mm}$ after closed reduction, open reduction and internal fixation is recommended. For the fracture with small displacement, conservative or surgical treatment is chosen, mainly based on the stability of the fracture, the existence of articular cartilage hinges determines the stability of LCF. ${ }^{9}$

Therefore, for micro-displaced fractures whose stability can not be completely judged by X-ray films, it is suggested that the integrity of articular cartilage hinges can be judged by elbow arthrography, ${ }^{10}$ or the articular surface can be detected by arthroscopy, so as to determine whether cartilage hinges are broken or not, so as to avoid missed diagnosis and mistreatment of micro-displaced LCF in children. ${ }^{9}$ There are also multicenter studies in China that LCF with displacement $\leq 2 \mathrm{~mm}$ is prone to re-displacement. Early percutaneous fixation is recommended to avoid complications such as open reduction, poor function and premature closure of epiphysis caused by fracture redisplacement. ${ }^{11}$

\section{Comparison of the selection of different internal fixation materials}

At present, for the LCF, internal fixation materials that need surgical treatment, the main choice is Kirschner wire, hollow or lag screw, absorbable screw and so on. The selection advantage of Kirschner needle is that it is easy to operate and easy to take out, and even the Kirschner needle outside the skin can be taken out in the outpatient clinic, and there is no need for special tools. And the advantages of Kirschner wire fixation include the ability to fix smaller epiphysis or reduce the risk of epiphyseal injury. ${ }^{12}$ However, the debate on Kirschner wire fixation is mainly focused on whether the Kirschner wire should be kept outside the skin. Some studies have found that there is no significant difference in the subcutaneous infection rate of whether the Kirschner wire is embedded or not, but the skin erosion rate of the buried 
subcutaneous group is higher, so it is suggested that it should not be routinely buried subcutaneously in order to save more economic costs. ${ }^{13}$

As for the scholars who choose screw fixation, they think that screw fixation is more stable in biomechanics than Kirschner wire fixation. ${ }^{14} \mathrm{Li}$ et al. ${ }^{15}$ comparison of Kirschner wire and cannulated screw internal fixation can effectively treat displaced LCF, there is no statistical difference between the two clinical outcomes, but Kirschner needle will occur surrounding skin infection. Das et al. ${ }^{16}$ reported that the infection rate of percutaneous fixation of Kirschner needle ranged from 2-9.8\%, and the subcutaneous infection rate of buried skin ranged from $0.5-2 \%$. Due to the deep placement of screws, it rarely irritates the skin, so the infection rate is very low. However, compared with the Kirschner wire that can penetrate the head epiphysis, Gilbert et al ${ }^{17}$ suggested that screws are usually placed through the metaphysis of the lateral condyle, rather than through the ossification nucleus of the capitulum humeri, to reduce the risk of re-injury of the epiphysis.

III. Comparison of postoperative complications and functional recovery.

The postoperative complications of LCF included bone nonunion, ischemic necrosis, premature fusion of epiphysis, overgrowth of lateral condyle, stiffness, cubitus varus or cubitus valgus and fish tail deformity. ${ }^{18}$ Launay et al. ${ }^{19}$ calculated that the operative complications after Kirschner wire fixation included pin site infection, lateral overgrowth and loss of fixation, while fixation failure or loss of position led to fracture displacement and reoperation or nonunion, which was a serious complication of LCF. Pace et al. ${ }^{20}$ found that the overall incidence of bone nonunion after LCF was $1.4 \%$, and the only important risk factor for bone nonunion was type III fracture. Compared with Kirschner wire fixation, screw fixation provides stronger biomechanical stability, and the incidence of lateral overgrowth, loss of fixation and infection is relatively low. However, Shirley et al. ${ }^{12}$ also pointed out that the results of screw fixation and the subsequent risk of iatrogenic epiphyseal injury have not been well determined, so early epiphyseal closure and growth arrest caused by screws are still noteworthy.

Foreign studies have found that $4 \%$ of the patients with LCF screw fixation have the phenomenon of overflexion or loss of activity, and there is no growth stagnation or change of lifting angle. Long-term follow-up showed that the average extension loss was $2^{\circ}\left(\right.$ range $\left.0{ }^{\circ} 25^{\circ}\right)$ and the average flexion loss was $8^{\circ}$ (range $\left.0^{\circ} 25^{\circ}\right) .{ }^{12}$ The growth disorder of the epiphysis of the distal humerus may affect the carrying angle and lead to cubitus valgus or varus deformity. In some cases, the hypertrophy of the posterolateral condyle of the fracture can increase the Baumann angle, leading to cubitus varus deformity, while growth stagnation reduces the Baumann angle, leading to cubitus valgus deformity. ${ }^{21}$

IV. about the postoperative nursing and the timing of internal fixation

Whether closed or open reduction, internal fixation with Kirschner wire or screw fixation, scholars at home and abroad seem to agree that postoperative fixation should be supplemented by plaster or brace. As for how long external fixation is appropriate, there is no unified standard. Li et al. ${ }^{15}$ think that Kirschner wire 
fixation requires longer external fixation and local skin care. Gilbert et al. ${ }^{17}$ believe that screw fixation can appropriately reduce the time of plaster external fixation, which is more conducive to postoperative exercise of elbow function and a wide range of joint motion. The external fixation time in the screw fixation group was also shorter than that in the Kirschner wire group. Early removal of external fixation and functional exercise were considered to avoid joint stiffness.

There is no uniform standard for how long to take internal fixation after LCF, and considering that intraarticular fracture healing may take longer, Cardona et al. ${ }^{22}$ suggested that the time of internal fixation ranges from 3 to 8 weeks. Raghavan et al. ${ }^{23}$ believe that buried Kirschner wires can allow indwelling longer, thus promoting better fracture healing, while exposure to the skin seems to need to be removed earlier, mainly to reduce the risk of pin tract infection. More studies are based on the time of fracture healing, and some studies have found that fractures heal within 6 weeks clinically and radiographically, so it is recommended that the average time of internal fixation is 6 weeks or more. ${ }^{22}$ Another study found that the average healing time in the Kirschner wire group was 9.6 weeks, and the average healing time of all fractures after screw fixation was 7.8 weeks. ${ }^{17}$

\section{Some focus and prospects on LCF}

At present, about the clinical prognosis of Kirschner wire or screw fixation, most of the literature data show that there is no statistical difference. Whether the Kirschner wire is buried under the skin and whether the screw can penetrate the epiphysis of capitulum humeri, the length of fixation time is not final. Due to the bleeding and swelling after soft tissue tear, the infiltration of synovial fluid, the stimulation of internal fixation and other factors, incision infection can not be completely controlled, especially the incision scar caused by open reduction and the need for secondary internal fixation. it has been reported that the use of absorbable screws for fracture fixation will overcome the shortcomings of metal screw fixation which need to be removed by second operation. At the same time, it still retains the advantage of biomechanical stability. ${ }^{24}$ It has also been reported that arthroscopy can be used to assist the displacement of humeral lateral condyle fractures larger than $2 \mathrm{~mm}$ to achieve closed reduction and percutaneous needle puncture. ${ }^{25}$ In the case of ischemic necrosis of the lateral condyle (avascularnecrosis,AVN) after LCF, Conaway et al. [26] believed that the occurrence of AVN was mainly due to excessive peeling of the soft tissue of the lateral condylar fracture, rather than the approach used, and recommended that the posterior approach did not need to peel off the soft tissue, and still had a good visibility of fracture reduction.

\section{Declarations}

Ethics approval and consent to participate

Not applicable 
Consent for publication

Yes

Availability of data and materials

Yes

Competing interests

The authors declare that they have no competing interests

Funding

This study was supported by Henan Provincial Medical Outstanding Talent Foundation, Henan Provincial Medical Youth Talent Foundation.

Authors' contributions

1. Objective: WANG Jia-xiang. 2. Study selection: LUO Xian-yong and WANG Jia-xiang. 3.Data sources, Data extraction and Data synthesis: LUO Xian-yong and CHEN Xin-rang. 4. Conclusions: WANG Jia-xiang. 5. Wrote the manuscript: LUO Xian-yong.The author (s) read and approved the final manuscript.

\section{Acknowledgements}

This study was supported by Henan Provincial Medical Outstanding Talent Foundation, Henan Provincial Medical Youth Talent Foundation.

We thank the patients who volunteered to provide case data for this study.

\section{References}

1. Morrisey RT, Weinstein SL. Lovell and Winter's pediatric orthopaedics, 6th edition. Philadelphia, PA: Lippincott, Williams, and Wilkins. 2006, 2:pp. 1454-1457.

2. Jakob R, Fowles JV, Rang M. et a1. Observations concerning fractures of the lateral humeral condyle in children[J]. J Bone Joint surg Br. 1975;57(4):430-6.

3. Dhillon KS, Sengupta S, Singh BJ. Delayed management of fracture of the lateral humeral condyle in children[J]. Acta Orthop Scand. 1988;59(4):419-24. 
4. Milch H. Fractures and fracture dislocations of the humeral condyles[J]. J Trauma. 1964;4:592-607.

5. Pirker ME, Weinberg AM, Hollwarth ME, et al. Subsequent displacement of initially nondisplaced and minimally displaced fractures of the lateral humeral condyle in children[J]. J Trauma. 2005;58(6):1202-7.

6. H.Sharma, K.Chirputkar, R.D.D.Duncan. Management of lateral humeral condylar mass fractures in children[J]. Current Orthopaedics, 2007,21(2): 145-149.

7. Nazareth A, VandenBerg CD, Sarkisova N, et al. Prospective Evaluation of a Treatment Protocol Based on Fracture Displacement for PediatricLateral Condyle Humerus Fractures: A Preliminary Study[J]. J Pediatr Orthop. 2019, 00:000-0.

8. Song KS, Kang $\mathrm{CH}$, Min BW, et al. Closed reduction and internal fixation of displaced unstable lateral condylar fractures of the humerus in children[J]. J Bone Joint Surg Am. 2008;90(12):2673-81.

9. Temporin K, Namba J, Okamoto M, et al. Diagnostic arthroscopy in the treatment of minimally displaced lateral humeral condyle fracturesin children[J]. Orthop Traumatol Surg Res. 2015;101(5):593-6.

10. Abzug JM, Dua K, Kozin SH, et al. Current Concepts in the Treatment of Lateral Condyle Fractures in Children[J]. J Am Acad Orthop Surg. 2020;28(1):9-19.

11. Ze Renhao L, Jin M, Haibo, et al. Surgery versus conservative treatment for displaced $\leq 2 \mathrm{~mm}$ fractures of lateral humeral condyle: a multi-center retrospective study[J]. Chin J Pediatr Surg. 2016;37(12):909-12.

12. Shirley E, Anderson M, Neal K, et al. Screw Fixation of Lateral Condyle Fractures: Results of Treatment[J]. J Pediatr Orthop. 2015;35(8):821-4.

13. Ormsby NM, Walton RD, Robinson S, et al. Buried versus unburied Kirschner wires in the management of paediatric lateral condyle elbowfractures: a comparative study from a tertiary centre[J]. J Pediatr Orthop B. 2016;25(1):69-73.

14. Schlitz RS, Schwertz JM, Eberhardt AW, et al. Biomechanical Analysis of Screws Versus K-Wires for Lateral Humeral Condyle Fractures[J]. J Pediatr Orthop. 2015;35(8):93-7.

15. Li WC, Xu RJ. Comparison of Kirschner wires and AO cannulated screw internal fixation for displaced lateralhumeral condyle fracture in children[J]. Int Orthop. 2012;36(6):1261-6.

16. Das De S, Bae DS, Waters PM. Displaced humeral lateral condyle fractures in children: should we bury the pins[J]? J Pediatr Orthop. 2012;32(6):573-8.

17. Gilbert SR, MacLennan PA, Schlitz RS, et al. Screw versus pin fixation with open reduction of pediatric lateral condyle fractures[J]. J Pediatr Orthop B. 2016;25(2):148-52.

18. Weiss JM, Graves S, Yang S, et al. A new classification system predictive of complications in surgically treated pediatric humeral lateral condyle fractures[J]. J Pediatr Orthop. 2009;29(6):602-5.

19. Launay F, Leet Al, Jacopin S, et al. Lateral humeral condyle fractures in children: a comparison of two approaches to treatment[J]. J Pediatr Orthop. 2004;24(4):385-91. 
20. Pace JL, Arkader A, Sousa T, et al. Incidence, Risk Factors, and Definition for Nonunion in Pediatric Lateral Condyle Fractures[J]. J Pediatr Orthop. 2018;38(5):257-61.

21. Yilmaz E, Karakurt L, Belhan $O$, et al. Variation of carrying angle with age, sex, and special reference to side[J]. Orthopedics. 2005;28(11):1360-3.

22. Cardona JI, Riddle E, Kumar SJ. Displaced fractures of the lateral humeral condyle: criteria for implant removal[J]. J Pediatr Orthop. 2002;22(2):194-7.

23. Raghavan R, Jones A, Dwyer AJ. Should Kirschner wires for fixation of lateral humeral condyle fractures in children be buried or left exposed? A systematic review[J]. Orthop Traumatol Surg Res. 2019;105(4):739-45.

24. Su Y, Chen K, Qin J. Retrospective study of open reduction and internal fixation of lateral humeral condyle fractureswith absorbable screws and absorbable sutures in children[J]. Medicine. 2019;98(44):17850.

25. Kang MS, Alfadhil RA, Park SS. Outcomes of Arthroscopy-assisted Closed Reduction and Percutaneous Pinning for a Displaced Pediatric Lateral Condylar Humeral Fracture[J]. J Pediatr Orthop. 2019;39(7):e548-51.

26. Conaway WK, Hennrikus WL, Ravanbakhsh S, et al. Surgical treatment of displaced pediatric lateral condyle fractures of the humerus by the posterior approach[J]. J Pediatr Orthop B. 2018;27(2):12833.

\section{Figures}
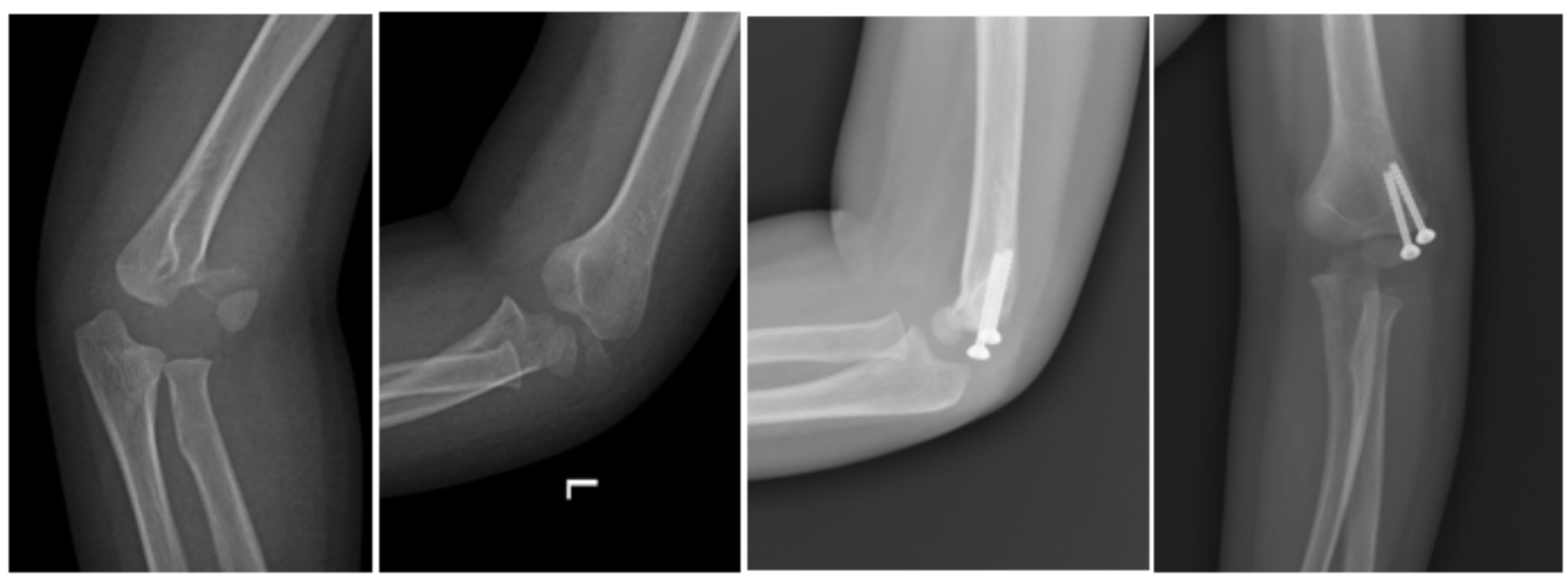

\section{Figure 1}

male, 5 years old, JacobIII type, with open reduction and cannulated cancellus screw fixation. 

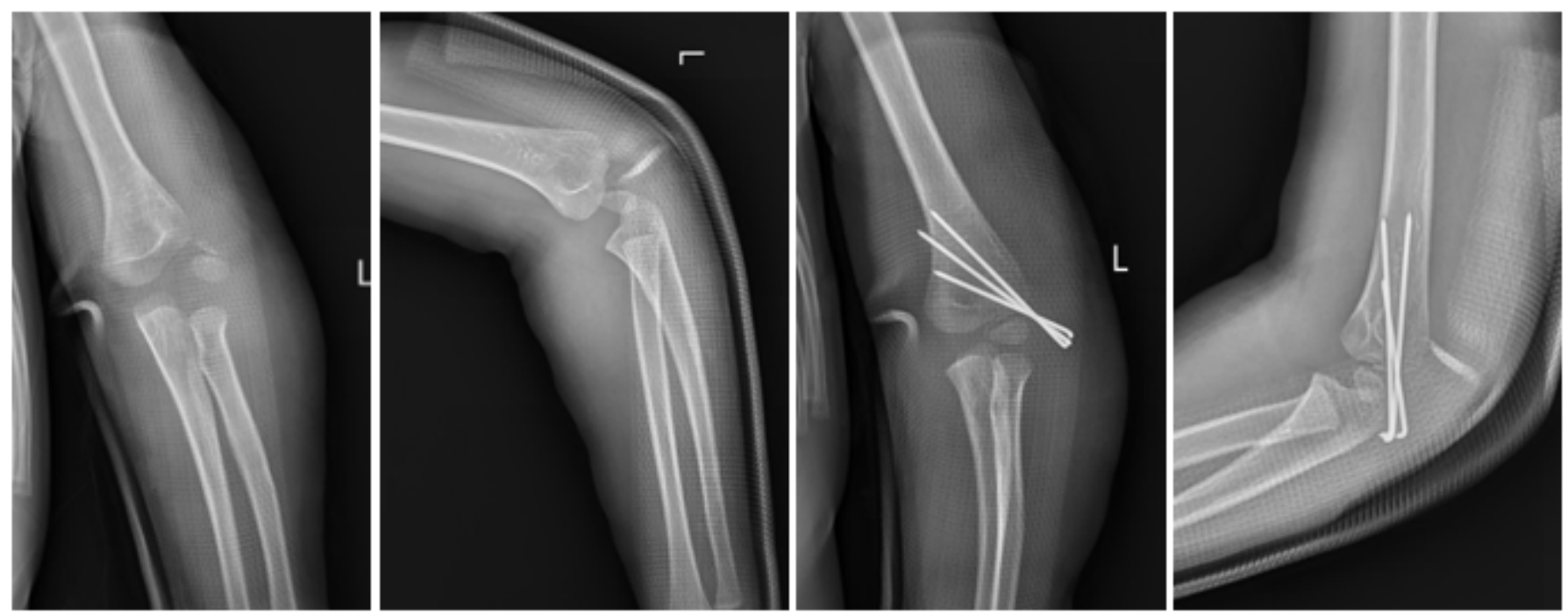

Figure 2

male, 4 years old, Jacoblll type, with open reduction and Kirschner wire fixation. 